\title{
Clinical and Laboratory Profiles of Children with West Syndrome: Experience of 50 Cases in a Tertiary Hospital in Bangladesh
}

\author{
MAHUA CHANDRA ${ }^{1}$, NARAYAN SAHA ${ }^{2}$, SK AZIMUL HOQUE ${ }^{3}$, PROVAT KUMAR SARKAR ${ }^{4}$, \\ SHAMEEM ARA BEGUM ${ }^{5}$, BANITAMISTRY ${ }^{5}$
}

\begin{abstract}
Background: West Syndrome (WS) consists of epileptic spasms, hypsarrhythmia on EEG and psychomotor delay or regression in children.

Objective: To observe the clinical and laboratory profiles of children with west syndrome.

Materials \& Methods: This was a cross sectional study and done from July 2017 to June 2018 in Pediatric Neurology OPD, NINS among total 50 cases of WS (age of $>$ 2 month to 2 years). WS was diagnosed by direct observation or video recording of spasm, along with history of developmental delay or regression and EEG change. Collected data regarding demography, detailed of spasm, clinical examination, radiological and EEG findings were analyzed.
\end{abstract}

Results: Mean age of the patients was $12.61 \pm 7.11$ months. Males (58.0\%) were predominant than females (42.0\%). Mean age of onset of spasm was $5.88 \pm 3.73$ months. Type of spasm: Flexor in $88.0 \%$ cases, extensor in $6.0 \%$ cases, mixed in $4.0 \%$ and asymmetric in $2.0 \%$. Microcephaly was present in $90.0 \%$ cases and in $64 \%$ cases H/o perinatal asphyxia (PNA) was present. In EEG, Hypsarrhythmia was found in $62.0 \%$ cases and modified hypsarrhythmia in $38.0 \%$ cases. In CT scan of brain, cerebral atrophy was found in $62.5 .0 \%$ cases, stroke in $6.0 \%$ cases. Out of all WS patients $82 \%$ were symptomatic and $18 \%$ were either cryptogenic or idiopathic.

Conclusion: Flexortype spasm was found more commonly in West syndrome. History of perinatal asphyxia (PNA) was present in majority of cases. In EEG findings, hypsarrhythmia was found more than modified hypsarrhythmia.

Key words: West syndrome (WS), Perinatal asphyxia (PNA), Developmental delay, Epileptic spasm, Hypsarrhythmia.

\section{Introduction}

West syndrome (WS) is a rare form of epilepsy that begins during early infancy characterized by three features namely, epileptic spasms, developmental delay, and characteristic electroencephalogram (EEG)

1. Junior consultant, Dept. of Pediatric Neurology, NINS.

2. Professor and Head, Department of Pediatric Neurology, NINS

3. Associate Professor, Department of Pediatric Neurology, NINS

4. Assistant Professor, Department of Neurology, NINS

5. Assistant Professor, Dept. of Pediatric Neurology, NINS

Correspondence: Dr Mahua Chandra, Junior consultant, Department of Pediatric Neurology, National Institute of Neuroscience and Hospital, Dhaka. Cell no +8801711022930; Email: chandra.mahua@yahoo.com

Received: 1/02/2020

Accepted: $15 / 10 / 2020$ pattern called hypsarryhythmia. ${ }^{1}$ The peak age of onset of WS is 4-6 months, the incidence of WS is $2-3$ cases per 10,000 live births, ${ }^{2,3}$ and a lifetime prevalence rate of $1.5-2$ per 10,000 children. ${ }^{4}$ There appears to be a slightly higher incidence rate in males than in females. ${ }^{5}$ Dr. William James West first described the syndrome in 1841, when his son was affected. ${ }^{6}$ WS is classified as follows: 1 ) Symptomatic: with identifiable prenatal, perinatal, and postnatal causes with developmental delay at the presentation time 2) Cryptogenic: unknown underlying cause, normal development at the onset of spasms, normal neurological exam and neuroimaging, and no abnormality in the metabolic evaluation; 3) Idiopathic: 
pure functional cerebral dysfunction with complete recovery, no residual dysfunction, normal neuroimaging and normal etiologic evaluation, and normal neurodevelopment. ${ }^{7}$

The etiologies of WS are often classified into prenatal, perinatal and postnatal groups. Prenatal etiologies include CNS malformations (focal cortical dysplasia, lissencephaly, holoprosencephaly, hemimegalencephaly, callosal agenesis/Aicardi syndrome), chromosomal abnormalities (trisomy 21, Miller-Dieker syndrome), single-gene errors, neurocutaneous syndrome (TS, NF1, incontinentia pigmenti), congenital central nervous system infections (TORCH), and rarely, in-born error of metabolism. Perinatal causes include hypoxic ischemic encephalopathy and hypoglycemia and postnatal factors include intracranial infections, hypoxic-ischemic insults and brain tumors. ${ }^{7}$

Epileptic spasms in WS having a typical EEG patterns. ${ }^{8}$ This spasm can be divided mainly into three types (flexor, extensor, and mixed flexor-extensor spasms), and they can also be asymmetrical. ${ }^{9}$ This study was done to observe the clinical and laboratory profile of WS patients in a tertiary hospital in Bangladesh.

\section{Materials \& Methods}

This cross sectional study was done in the Outpatient and inpatient department of Pediatric Neurology, National Institute of Neuroscience and Hospital (NINS\& H), Sher-E-Bangla Nagar, Dhaka from July 2017 to June 2018. After taking informed written consent from parents or caregiver children within $>2$ month to 2 years age group with West Syndrome (WS) were included in this study. WS was diagnosed by history of presence of clinical spasm that occur mainly in clusters either flexor, extensor or both or asymmetric by direct observation or video recording along with history of developmental delay or regression, and EEG demonstrating hypsarrhythmia or modified hypsarrhythmia.

Children were evaluated thereafter through detail history and clinical examination. History related to seizure type, frequency, age at onset, antenatal, natal and postnatal details, family history, developmental history and the ongoing treatment were noted. Through clinical examinations were done. Base line CT scan of brain and EEG were done in all case and findings were noted. Qualitative data were expressed as frequency \& percentage and quantitative data were expressed as mean \& standard deviation. Collected data were processed and analyzed using software Statistical Package for Social Science (SPSS) version 20.0. 95\% confidence interval was calculated. Mann-Whitney $U$ test, Chi-square test and Wilcoxon signed ranks test and unpaired $t$ test were done to see the level of significance. A value of $p<0.05$ was considered statistically significant for all test.

\section{Results}

In this study on West syndrome mean age of the patients was $12.61 \pm 7.11$ months. Males (58.0\%) were predominant than females $(42.0 \%)$. Mean age of onset of spasm $5.88 \pm 3.73$ months. Type of spasm: Flexor in $88.0 \%$ cases, extensor in $6.0 \%$ cases, mixed in $4.0 \%$ and asymmetric in $2.0 \%$. History of perinatal asphyxia was observed in $64.0 \%$ cases. Developmental delay was present in $82 \%$ cases. Consanguinity was present in $6.0 \%$ cases and microcephaly was found in $90.0 \%$ cases. Majority of the patients (27\%) had 6-10 cluster / day and mean cluster of spasm per day was $8.27 \pm$ 4.34. Perinatal asphyxia was present in $64.0 \%$ cases, neonatal septicemia was present in $8.0 \%$, meningitis was present in $4.0 \%$ cases and unknown etiology was evident in $24.0 \%$ cases. Majority of patients $(82 \%)$ were symptomatic and $18 \%$ were either cryptogenic or idiopathic group. Hypsarrhythmia was found in $62.0 \%$ cases and modified hypsarrhythmia was present in $38.0 \%$ cases. Cerebral atrophy was found in $62 \%$ cases, Ischemic change in $6.0 \%$ cases.

Table I

Demographic profile of the patients $(n=50)$

\begin{tabular}{lcc}
\hline & Frequency $(\mathrm{n})$ & $(\%)$ \\
\hline Age (months) & \multicolumn{2}{c}{} \\
$\quad 1-12$ & 29 & 58.0 \\
$13-24$ & 21 & 42.0 \\
Mean \pm SD & \multicolumn{2}{c}{$12.61 \pm 7.11$} \\
Gender & \multicolumn{2}{c}{} \\
$\quad$ Male & 29 & 58.0 \\
Female & 21 & 42.0 \\
\hline
\end{tabular}


Table II

Clinical characteristics of the patients $(n=50)$

\begin{tabular}{lcc}
\hline History & Frequency $(\mathrm{n})$ & $(\%)$ \\
\hline Age of onset of spasm & $5.88 \pm 3.73$ & \\
(months) & & \\
Type of spasm & & \\
Flexor & 44 & 88.0 \\
Extensor & 3 & 6.0 \\
Mixed & 2 & 4.0 \\
Asymmetric & 1 & 2.0 \\
History of perinatal asphyxia & 32 & 64.0 \\
Development history before spasm & \\
Age appropriate & 9 & 18.0 \\
Delayed & 41 & 82.0 \\
Consanguinity & 3 & 6.0 \\
Microcephaly present & 45 & 90.0 \\
\hline
\end{tabular}

Table III

Cluster of spasms of study patients

\begin{tabular}{lc}
\hline Cluster of spasm/day & Before Rx \\
\hline$>20$ & $1(2 \%)$ \\
$16-20$ & $3(6 \%)$ \\
$11-15$ & $3(6 \%)$ \\
$6-10$ & $27(54 \%)$ \\
$0-5$ & $16(32 \%)$ \\
Mean \pm SD & $8.27 \pm 4.34$ \\
\hline
\end{tabular}

Table IV

Antecedent illness of the study patients $(n=50)$

\begin{tabular}{lc}
\hline Etiology & Frequency \\
\hline Perinatal asphyxia & $32(64 \%)$ \\
Neonatal septicemia & $4(8 \%)$ \\
Meningitis & $2(4 \%)$ \\
Unknown & $12(24 \%)$ \\
\hline
\end{tabular}

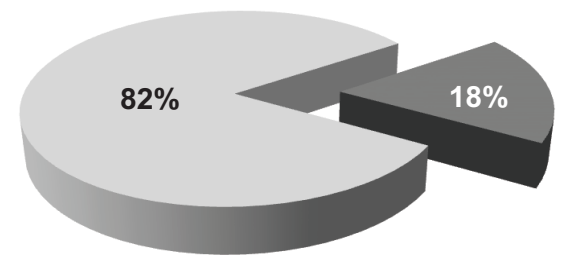

Symptomatic

Cryptogenic/Idiopathic

Fig.-1: Classification of study patients of West syndrome $(n-50)$

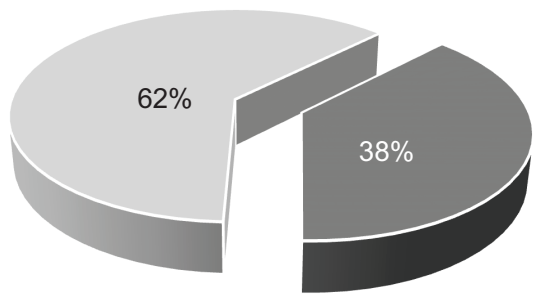

Hypsarrhythmia

Modified hypsarrhythmia

Fig.-2: Pie chart of EEG findings of the patients

Table V

Findings of CT scan of brain of the patients $(n=50)$

\begin{tabular}{lc}
\hline CT findings & Frequency (\%) \\
\hline Normal & $14(28 \%)$ \\
Cerebral atrophy & $31(62 \%)$ \\
Ischemic change & $3(6 \%)$ \\
Hydrocephalus & $1(2 \%)$ \\
Subdural effusion & $1(2 \%)$ \\
\hline
\end{tabular}

Discussion:

In this cross sectional study on West syndrome mean age of the patient was $12.87 \pm 7.15$ months. Fusco et al. ${ }^{9}$ showed mean age of presentation was 9.4 months that was lower age than this study and Kaushik et al. ${ }^{11}$ found 13.1 months that finding was nearly consistent with this study. It was found that among study patients male were $29 / 50(55.8 \%)$ and female were $21 / 50(44.2 \%$ ). Male preponderance $72 \%$ and $81 \%$ respectively that was also found in similar studies. ${ }^{10,11}$ The reason of male preponderance may be due to more attention of family members to treat male babies and genetic etiology. ${ }^{12}$

In this study some important clinical variables were evaluated. Among them we observed mean age of onset of infantile spasms $7.88 \pm 1.99$ months. The age of onset of spasm in this study revealed slight higher than other studies done by Khreisat HW and Kaushik et al. 10,11 One study found age onset of spasms was ranged from 1 month to 30 months mean 5.3 months (4.6 SD) and another showed that age ranged from 1 month to 1 year and 6 months, (mean 4.8 months). We also observed that most common type of spasm was flexor spasm 44/50 (88.0\%) which was comparable to other studies who found majority of patient had flexor spasms $52 \%$ and $76 \%$ 
respectively. ${ }^{10,11}$ In this study it was found that $32 /$ $50(66 \%)$ patient had history of PNA. However that was not similar to study done by Khreisat $\mathrm{HW}^{10}$ where they found history of PNA was present in $48 \%$. This high number of PNA may be explained by poor socioeconomic background, poor delivery service. Consanguinity was present in $6 \%$ of cases. In this study most of the patients had $\mathrm{h} / \mathrm{o}$ developmental delay $41 / 50(82.0 \%)$ before spasm presentations and microcephaly $45 / 50(90 \%)$ was the commonest associated problem was supported by another study in Jordan. They found developmental delay prior to onset of spasms in $52 \%$ and microcephaly ( $26 \%$ ) was the commonest associated problem. ${ }^{10}$

In our study in initial EEG hypsarrythmia was found $31 / 50(62 \%)$ cases and modified hypsarrthymia in 19/ $50(38 \%)$ cases. In an African study Keshave A et al. ${ }^{13}$ also found modified hypsarrhythmia in $37.5 \%$ cases and classic hypsarrhythmia in $62.5 \%$ cases in initial EEG findings which is similar to this study. Regarding number of cluster, majority of the patients (27\%) had 6-10 cluster/day and mean cluster of spasm per day was $8.27 \pm 4.34$. In an African and in another Indian study showed average 5 and 12.4 (8.8) cluster of spasms per day respectively. ${ }^{11,13}$

CT scan of brain were done and found that $72 \%$ patient had brain abnormalities. From which $62 \%$ patient had cerebral atrophy. The abnormal imaging was more in number in comparison to another study done in Jordan that revealed imaging studies of the brain revealed abnormality in $40 \%$ patients. ${ }^{10}$ This high rate of abnormalities may be due to sequel of PNA which is high in our country. Regarding etiology PNA was present in $64.0 \%$ cases, neonatal septicaemia in $8.0 \%$, meningitis in $4.0 \%$ cases and unknown etiology in $24.0 \%$ cases among study patients. These findings matches with related study done by Khreisat HW. ${ }^{10}$ This study showed 41/50 (78.8 \%) cases were symptomatic and 9/50(17.3\%) cases were cryptogenic. Two other related studies also found that symptomatic cases are more $(78 \%$ and $64 \%$ ) which is similar to our observation. ${ }^{14,10}$

\section{Conclusion:}

This study demonstrates that males were predominant among the study subject of West Syndrome. Flexor type spasm was more common. History of perinatal asphyxia (PNA) was present in majority of the cases. In EEG findings hypsarrhythmia was common. In CT scan of brain cerebral atrophy was found in majority cases and most of the west syndrome patients were symptomatic.

Conflict of interest: There is no conflict of interest relevant to this paper to disclose.

Funding agency: This research project was not funded by any group or any institution.

\section{References}

1. Song JM, Hahn J, Kim SH, Chang MJ. Efficacy of Treatments for Infantile spasm: A Systematic Review. Clin Neuropharmacol 2017;40:63-84.

2. Riikonen R. Epidemiological data of West syndrome in Finland. Brain Dev 2001; 23: 539-41.

3. Ludvigsson P, Olafsson E, Sigurdardottir S, Hauser WA. Epidemiological features of infantile spasms in Iceland. Epilepsia1994; 35: 802-5.

4. Trevathan E, Murphy CC, Yeargin-Allsopp M. The descriptive epidemiology of infantile spasms among Atlanta children. Epilepsia1999; 40: 748-51.

5. Cowen LD, Hudson LS. The epidemiology and natural history of infantile spasms. J Child Neurol 1991; 6: 355-64.

6. West WJ. On a peculiar form of infantile convulsions. Letter to the editor. Lancet 1841; 1: 724-725.

7. Taghdiri MM, Nemati H. Infantile Spasm: A Review Article. Iran J Child Neurol. 2014; 8: 1-5.

8. Watanabe K, Negoro T, Okumura A. Symptomatology of infantile spasms. Brain Dev. 2001; 23:453-66.

9. Fusco L, Vigevano F. Ictal clinical electroencephalographic findings of spasms in West syndrome. Epilepsia. 1993; 34: 671-8.

10. Khreisat HW. Clinical Profile of Infants with Hypsarrhythmia. Acta Inform Med. 2011; 19: 149-152.

11. Kaushik JS, Patra B, Sharma S, Yadav D, Aneja S. Clinical spectrum and treatment outcome of West Syndrome in children from Northern India. Seizure 2013; 22:617-621.

12. Pavone $P$, Striano P, Falsaperla R, Pavone L, Ruggieri M. Infantile spasms syndrome, West syndrome and related phenotypes: what we know in 2013. Brain Dev. 2014; 36:739-51.

13. Keshave A, Yende-Zuma N, Mubaiwa L, M Adhikari. The clinical profile and outcome of children with West syndrome in KwaZulu-Natal Province, South Africa: A 10-year retrospective review. Afr. j. child health 2017; 11:135-40.

14. Kodama K, Omata T, Arai H, Tanabe Y. Study of the efficacy of low-dose synthetic ACTH therapy without tapering to treat West syndrome. 2016; 48(3):195-8 doi.org/10.11251/ojjscn.48.195. 